\title{
Chlorophyll fluorescence for classification of tomato fruits by their maturity stage
}

\author{
M. A. Abdelhamid ${ }^{1, *}, Y$ Y. A. Sudnik ${ }^{2}$, H. J. Alshinayyin ${ }^{3}$, and Fatma Shaaban ${ }^{1}$ \\ ${ }^{1}$ Agr. Eng. Dep., Faculty of Agriculture, Ain Shams University, Egypt. \\ ${ }^{2}$ Inst. of Eng. and energy, RSAU - Moscow Timiryazev Agri. Academy, Russia. \\ ${ }^{3}$ Ministry of Agriculture, Iraq.
}

\begin{abstract}
Classification of tomato fruit and control of post-harvest maturation based on the stage of maturity at harvesting, are necessary to ensure the highest possible quality and marketability of the final ripe product. A method of sorting tomatoes to assess the degree of their maturation based on the control of their chlorophyll fluorescence induction is proposed. Tomatoes (Black baron) at five different stages of maturity were used. Variance analysis (ANOVA) was performed and the Duncan's mean values were compared at a significance level of $p \leq 0.05$. In addition, the correlation between the chlorophyll fluorescence induction parameters and the maturity of the same fruit was carried out using the statistical software SPSS 20.0. The general pattern of fluorescence induction analysis was revealed; as the fruit matures, the value of both maximum fluorescence induction $(F m)$ and the coefficient of specific photosynthetic activity of tomatoes $(K f)$ decreases. Both $F m$ and $K f$ exhibited a statistically significant difference $(p \leq 0.05)$ between stages of maturity. A very strong negative correlation between slow chlorophyll fluorescence induction parameters ( $F m$ and $K f$ ) and maturity were observed. Moreover, there is a strong positive correlation between $\mathrm{Fm}$ and $\mathrm{Kf}$. In conclusion, the slow induction of chlorophyll fluorescence is a good indicator of the degree of maturity of tomato fruits and the proposed method had better reflected the actual ripening process of fruit per maturity stage.
\end{abstract}

\section{Introduction}

Tomato is one of the most important vegetable crops in the world due to its high consumption, year-round availability and high content of useful components. Tomato is an important supplier of nutrients such as folic acid, potassium, vitamins A and C, flavonoids, and carotenoids [1]. Black tomatoes are useful for preventing cancer. Unlike usual red tomatoes, black tomatoes contain more antioxidants that prevent the development of tumors. Black tomatoes have a beneficial effect on the cardiovascular system, strengthening it. And they can lower the blood pressure.

Black tomatoes contain high levels of vitamin $\mathrm{C}$ which is useful for overall strengthening of the body, supporting the immune system and protects against colds [2]. The maturity of a

\footnotetext{
* Corresponding author: Mahmoudabdelhamid@agr.asu.edu.eg
} 
tomato is one of the most important factors associated with evaluating its quality. The maturity assessment of tomatoes is necessary to establish the timing of fruit harvest, optimize storage conditions, forecast storage times, export, etc. [3]. Chlorophyll is an important pigment found in all plant tissues inside chloroplasts, it is imperative to provide energy demands of plant tissues required for growth. As the plastids differentiate into chloroplasts, the levels of chlorophyll become high enabling plant parts to have enhanced ability to absorb light. Consequently, the fruit attains its green mature state, then the chloroplast again differentiates into chromoplast or other types of protoplasts degrading chlorophyll. However, the chlorophyll content in the pericarp of red ripened tomato fruit is significantly reduced during ripening, it does not always disappear completely [4]. In particular, chlorophyll fluorescence is a tool for evaluating the quality characteristics of fruits, since it is a fast and easy-to-repeat method, suitable for using directly in the greenhouse. During the maturation of tomatoes, chlorophyll breaks down. Carotenoids, such as $\beta$-carotene and lycopene, accumulate in plastics as chloroplasts become chromoplasts. Thus, while green tomato fruits contain mainly chlorophyll, ripened fruits have the lowest level of chlorophyll [5]. This dynamic change in the composition and concentration of the pigment strongly affects optical parameters, such as reflection and fluorescence signals [6, 7]. Therefore, monitoring the level of chlorophyll in tomatoes is an important qualitative characteristic of their maturity. In a previous study [8] we observed the experimental changes in the concentration of chlorophyll on the degree of maturation of tomatoes of the " Alkacar " variety. The effects of changes in chlorophyll fluorescence concentration on fluorescence-based indices have been studied [9]. The internal characteristics of fruits such as fluorescence behavior of chlorophyll pigments can be used to determine fruit ripening. In turn, chlorophyll fluorescence provide another non-destructive and rapid technique that has been used to estimate the ripening of fruit and vegetables [10]. Therefore, the objective of this study was to classify tomato maturity using chlorophyll fluorescence analysis.

\section{Materials and methods}

\subsection{Plant material}

Tomato plants (Black baron) were grown in a greenhouse at Russian State Agricultural Academy named after K. A. Timiryazev, Russia. Tomato fruits were harvested at five different stages of maturity: mature green, blackish green, blackish yellow, blackish orange, reddish black.

\subsection{Chlorophyll fluorescence analysis}

For experimental studies, a device showed in Fig. 1 was developed [8] to assess the degree of maturation of tomatoes based on their chlorophyll fluorescence induction. The indicators of slow induction of chlorophyll fluorescence were determined based on the results of measurements in all groups of tomato fruits. The length of the fluorescence excitation wave was $470 \pm 8 \mathrm{~nm}$, and its intensity on the fetal surface varied from 3200 to $4700 \mathrm{mmol} . \mathrm{m}^{-2} \cdot \mathrm{s}^{-1}$. 


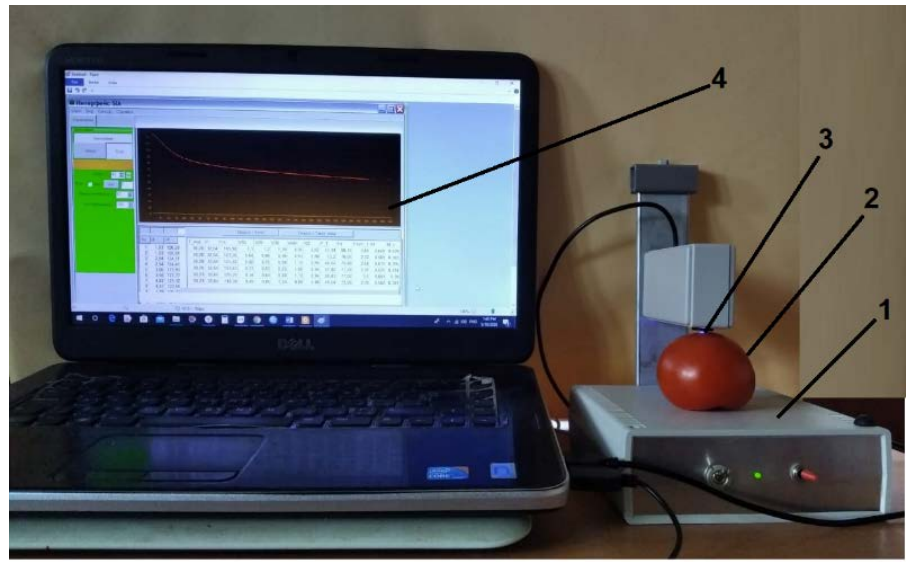

Fig. 1. Diagram of the system used in the present study. 1-device for measuring the level of slow induction of chlorophyll fluorescence; 2- tomato fruit under study; 3- radiation source (photodiode); 4computer with software.

The fluorescence of chlorophyll curve start with a sudden increase (Fig. 2), followed by a slow decrease in the intensity of induced fluorescence. This phenomenon was first described by Kautsky in 1931, to be named after "Kautsky effect". The fluorescence induction curve represents changes in the fluorescence emission of chlorophyll in a photosynthetic object. It can be divided into a fast phase that just takes around one second. This rapid phase includes reactions of the light phase of photosynthesis. In contrast, the slow phase may last for several minutes [11].

Slow induction of chlorophyll fluorescence based on the Kautsky curve result in many measurements showed in Fig. 2. These measurements include maximum of slow induction of chlorophyll fluorescence $(\mathrm{Fm})$, stationary levels of chlorophyll fluorescence $(\mathrm{Ft})$. And finally the coefficient of specific photosynthetic activity $(K f)$ that can be calculated according to the following equation: $K f=(F m-F t) / F m$. Slow induction of chlorophyll fluorescence was determined and analyzed using the Kautsky curve.

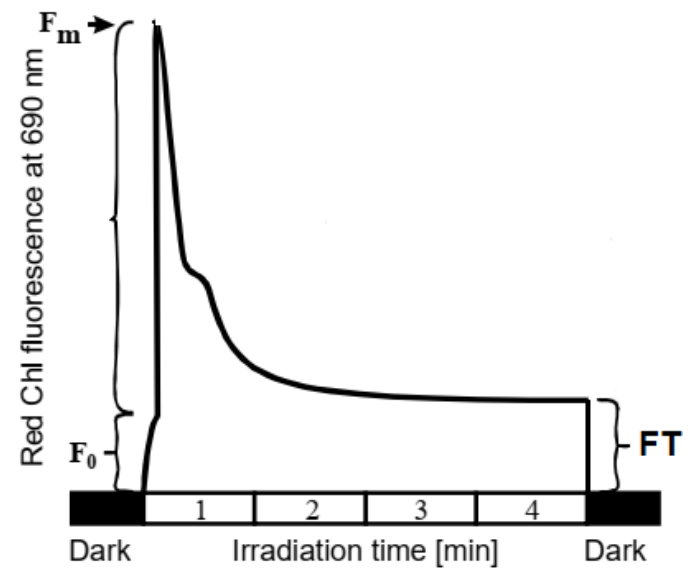

Fig. 2. Typical view of the chlorophyll fluorescence induction curve [12]. 


\subsection{Statistical analysis}

Data were plotted and analyzed using variance analysis (ANOVA), and the separation between averages were determined using the Duncan's multiple range test with a significance level of $\mathrm{p} \leq 0.05$. Correlation analysis between the selected parameters and the maturity of the same fruit was made by using Statistical Analysis Software SPSS (IBM statistics version 20.0, USA).

\section{Results and discussion}

The general pattern of chlorophyll fluorescence analysis in tomato (Black baron) was revealed. As the fruit becomes more mature, both $F m$ and $K f$ decreases. Green maturity stage (stage 1 ) is characterized by exhibiting the highest values of both $F m$ and $K f$. In contrary, the stage of full maturity (stage 5) is characterized by having the lowest values of these two parameters. Further sharp decline of the $\mathrm{Fm}$ and $\mathrm{K} f$ curve corresponds to low functional (photosynthetic) activity of the cells and the beginning of destructive processes. Thus, the lower the values of $F m$ and $K f$, the greater the maturity of tomato fruits. In addition, $F m$ and $K f$ found to be significantly different $(\mathrm{p} \leq 0.05)$ from maturation stage to another. During ripening, the green chlorophyll pigment is degraded, and carotenoids are synthesized. Carotenoids, in particular lycopene and $\beta$-carotene, are the main components of the pigmentation of ripened fruits in the pericarp of tomatoes and are responsible for the characteristic color of ripened tomatoes, giving them dark, red and orange colors, respectively. These carotenoids significantly influence the perception of the quality of fresh tomatoes [4]. In contrast to light of short wavelength in the case of using ultraviolet or laser, the chlorophyll fluorescence shows a quick rise that reach maximum level in just few seconds. After that, a process called fluorescence quenching occurs and includes the activation of both photochemical and non-photochemical processes. Consequently, a rapid reduction of the chlorophyll fluorescence is observed, after which a steady state is realized [13]. Also, primary photosynthetic reactions in the thylakoid membrane can be associated with light excited chlorophyll molecules in the photosystem II.

Although it has already been demonstrated that the chlorophyll fluorescence induction can be used to predict the maturity stage of mango [14], papaya fruit [15], lemons [16] and chicory seeds [17]. Our study proved that an accurate classification of tomato fruit according to the maturity stage could be achieved by using chlorophyll fluorescence induction.

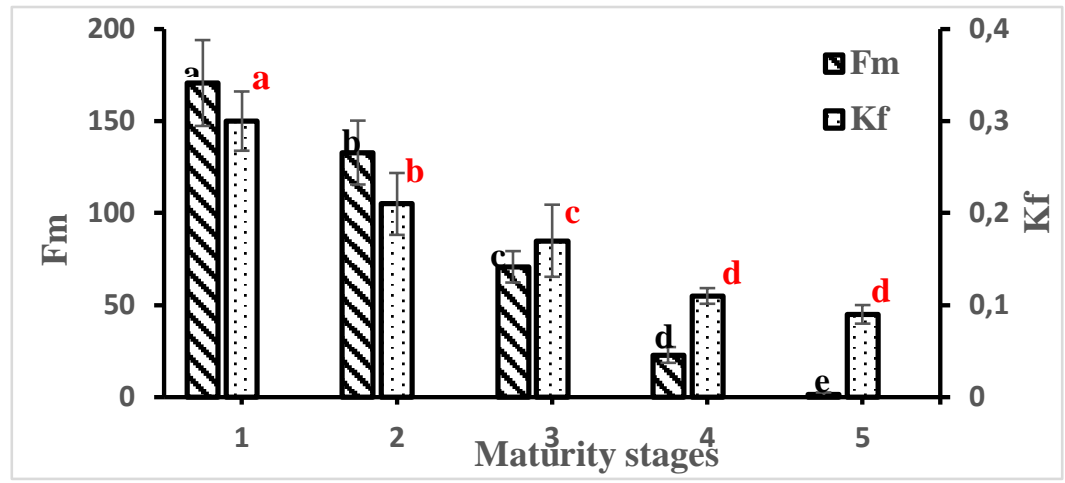

Fig. 3. Dependence of the maximum intensity of chlorophyll fluorescence $(F m)$ and the coefficient of specific photosynthetic activity $(K f)$ on the degree of maturity of tomatoes of the "Black baron" variety. Each bar represents the average value of specific maturity stage associated with standard error. The average values with different letters for each parameter differ significantly at $\mathrm{p} \leq 0.05$. 


\section{Correlations among variables}

Correlations between different measured variables were calculated to formulate the possible relationships between them (Table 1). Correlation analysis shows that both $\mathrm{Fm}$ and $\mathrm{Kf}$ parameters have a very strong negative relationship with maturity. This indicates that the slow induction of chlorophyll fluorescence is a better estimator of maturation in tomato fruits. The $F m$ and $K f$ exhibited a very strong positive relationship, since the first one $(F m)$ quantifies chlorophyll content of the fruit which is going to affect the photosynthetic activity by that fruit. [18] came up with an agreeably similar observation by showing that changes in fluorescence levels in apple fruits can be detected even without visual differences being apparent in the fruits studied. This put fluorescence measurements as an important tool in evaluating overall fruit quality and maturity.

Table 1. Correlation Coefficients between various variables measured during the experiment.

\begin{tabular}{|l|c|c|c|}
\hline & \multicolumn{1}{|c|}{ Maturity } & Fm & Kf \\
\hline Maturity & \multicolumn{1}{|c|}{-} & & \\
\hline Fm & $-0.955^{* *}$ & - & \\
\hline Kf & $-0.935^{* *}$ & $0.924^{* *}$ & - \\
\hline
\end{tabular}

\section{Conclusion}

This experiment was initiated with the objectives of determining chlorophyll fluorescence during the ripening of tomato fruits and its application as a nondestructive technique for determining fruit maturity. The maturation of tomatoes can be objectively assessed using the maximum slow induction of chlorophyll fluorescence $(F m)$ and coefficient of specific photosynthetic activity $(K f)$. The general pattern of chlorophyll florescence analysis in tomato (Black baron) was revealed. Both $F m$ and $K f$ decreases as the fruit matures. Comparing with other methods, chlorophyll florescence is less time-consuming and more accurate in determining the maturity of tomatoes by their color. Moreover, it allows for the separation of tomatoes based on maturation regardless of the maturated color.

Authors like to thank Prof. Budagovsky Andrey and Prof. Budagovskaya Olga (Academician of RAS Research Institute of Horticulture named after I. V. Michurin, Tambov, Russia) for helping us make the device. Authors also like to thank Mr. Ahmed S. Aly (TA, Fac. of Agric., Ain Shams Univ., Egypt) for helping us in writing the manuscript. Finally, we acknowledge the Egyptian Ministry of Higher Education for providing a scholarship to the first author during this study.

\section{References}

1. G. R. Beecher. Proceedings of the Society for Exper. Bio. and Med., 218 (2): (1998).

2. Pitaniye. Are black tomatoes good or bad?; 2020, May 15 Available from: https://pitaniye.com/pitanie/tomat/chernyj.html.

3. K. Choi, et al . Transactions of the ASAE. 38(1): p. 171-176 ( 1995).

4. $\quad$ N. Kozukue and M. Friedman. J. of the Sci. of Food and Agri., 83, 3 (2003).

5. $\quad$ G. Hobson and D. Grierson, Tomato, in Biochemistry of fruit ripening (Springer: Chapman and Hall, London, 1993).

6. C. Buschmann. Photosynthesis Research, 92, 2: p. 261-271(2007).

7. $\quad$ P. Chen and Z. Sun. J. of Agri. Eng. Research, 49: p. 85-98 (1991).

8. Y. A. Sudnik and M. A. Abdelhamid, Vestnik FSEIHPE "Moscow State Agroengineering University named after V.P. Goryachkin”,1 (95): p. 51-54 (2020).

9. T. Groher, et al. J. of the Sci. of Food and Agri.,98 (15): p. 5656-5665(2018).

10. A. Lai, et al. Postharvest Bio. and Tech., 43(3): p. 335-342 (2007). 
11. U. Schreiber, W. Bilger, and C. Neubauer, Chlorophyll fluorescence as a nonintrusive indicator for rapid assessment of in vivo photosynthesis, in Ecophysiology of photosynthesis (Springer, 1995).

12. H. Lichtenthaler, C. Buschmann, and M. Knapp. Photosynthetica, 43, 3 (2005).

13. E. Rosenqvist and O. van Kooten, Chlorophyll fluorescence: a general description and nomenclature, in Practical applications of chlorophyll fluorescence in plant biology ( Springer, 2003).

14. M. Lechaudel, L. Urban, and J. Joas. J. of agri. and food chem., 58,13 (2010).

15. I. U. Bron, et al. Postharvest Bio. and Tech., 33, 2: p. 163-173 (2004).

16. L. Nedbal et al. Photosynthetica, 38, 4: p. 571-579 (2000).

17. D. Ooms and M.-F. Destain . Biosystems Eng., 110, 2: p. 168-177(2011).

18. C. Huybrechts, T. Deckers, and R. Valcke. Predicting fruit quality and maturity of apples by fluorescence imaging: Effect of ethylene and AVG. in International Conference: Postharvest Unlimited 599. (2002). 